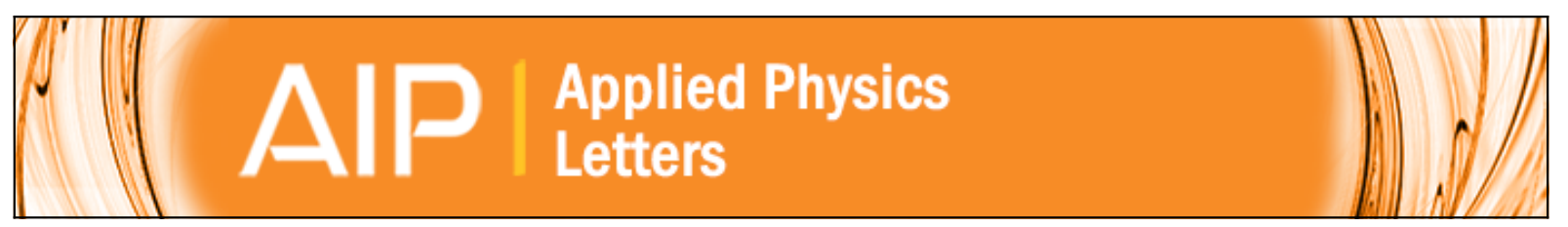

Ferromagnetism carried by highly delocalized hybrid states in Sc-doped ZnO thin films

Mohammed Benali Kanoun, Souraya Goumri-Said, Aurélien Manchon, and Udo Schwingenschlögl

Citation: Applied Physics Letters 100, 222406 (2012); doi: 10.1063/1.4721807

View online: http://dx.doi.org/10.1063/1.4721807

View Table of Contents: http://scitation.aip.org/content/aip/journal/apl/100/22?ver=pdfcov

Published by the AIP Publishing 


\title{
Ferromagnetism carried by highly delocalized hybrid states in Sc-doped ZnO thin films
}

\author{
Mohammed Benali Kanoun, ${ }^{\text {a) }}$ Souraya Goumri-Said, ${ }^{\text {b) }}$ Aurélien Manchon, \\ and Udo Schwingenschlögl ${ }^{\mathrm{c})}$ \\ PSE Division, KAUST, Thuwal 23955-6900, Saudi Arabia
}

(Received 1 April 2012; accepted 9 May 2012; published online 29 May 2012)

\begin{abstract}
We present first-principles results for Sc-doped $\mathrm{ZnO}$ thin films. Neighboring Sc atoms in the surface and/or subsurface layers are found to be coupled ferromagnetically, where only two of the possible configurations induce spin polarization. In the first configuration, the polarization is carried by the Sc $d$ states as expected for transition metal doping. However, there is a second configuration which is energetically favorable. It is governed by polarized hybrid states of the $\mathrm{Zn} s$, $\mathrm{O} p$, and Sc $d$ orbitals. Such highly delocalized states can be an important ingredient for understanding the magnetism of doped $\mathrm{ZnO}$ thin films. (C) 2012 American Institute of Physics. [http://dx.doi.org/10.1063/1.4721807]
\end{abstract}

The manipulation of the electronic spin for information processing gives rise to many new applications. However, the field of spintronics is in need of semiconducting ferromagnetic materials at room temperature. ${ }^{1}$ Nowadays, it is possible to achieve both properties in a single material by doping of magnetic impurities (e.g., V, Cr, Mn, Fe, Co or $\mathrm{Ni}$ ) into a semiconducting host (e.g., $\mathrm{ZnO}, \mathrm{TiO}_{2}, \mathrm{GaN}$ ), what is most commonly known as a dilute magnetic semiconductor (DMS) or dilute magnetic oxide (DMO). Of all DMOs, $\mathrm{ZnO}$ (owing to the wide band gap of $3.3 \mathrm{eV}$ and large exciton binding energy of $60 \mathrm{meV}$ ) is a potential host material for high Curie temperature ferromagnetism under doping with $3 d$ transition metal ions. ${ }^{2-4,6}$ Following the prediction of Dietl et al. ${ }^{5}$ that $\mathrm{ZnO}$ should have a high Curie temperature for hole doping, there have been various experimental reports proving or disproving a high Curie temperature ferromagnetism in this system. Theoretically, most of the materials that have been studied are semiconductors doped with transition metals. Because of the strong coupling between $3 d$ states of the magnetic ions and the host $p$ states, DMOs have been predicted and, in some cases, observed to show holemediated ferromagnetism above room temperatures. ${ }^{7,8}$ However, there is still a debate on whether the magnetic behavior of DMOs is an intrinsic property or due to nanoclusters of a magnetic phase or both.

A way to avoid problems with magnetic precipitates is to dope semiconductors or oxides with nonmagnetic elements instead of magnetic transition metals. Following this idea, room temperature ferromagnetism has been demonstrated in $\mathrm{Cu}$ and Ti-doped $\mathrm{ZnO} .^{9-12}$ Surprisingly, it has been observed that a (110) oriented thin film of $\mathrm{ZnO}$ with $\mathrm{Sc}$ impurities also shows ferromagnetism at room temperature ${ }^{13}$ with a Sc magnetic moment of $0.3 \mu_{B}$. It has been claimed that the fabrication of DMOs with nonmagnetic dopants is more challenging. On the theoretical side, there have been few reports on Sc-doped ZnO. Previous ab-initio calculations ${ }^{14}$ on bulk Sc-doped $\mathrm{ZnO}$ found no magnetic order, in

\footnotetext{
a)Mohammed.Kanoun@kaust.edu.sa.

b)Souraya.Goumri-Said@kaust.edu.sa.

${ }^{\text {c)} U d o . S c h w i n g e n s c h l o g l @ k a u s t . e d u . s a . ~}$
}

contrast to the experiment, which calls for an explanation. In contrast to the bulk, the broken periodicity at a surface leads to modifications of the chemical bonding. This particularly affects the orbitals which are no longer involved in bonding. Their energies will shift and they will consequently be populated more or less than in the bulk. If orbitals become partially occupied (ideally half filled), they may favor spin polarization.

In order to provide fundamental insight into the interaction of Sc with a $\mathrm{ZnO}$ host and how the interaction can induce magnetism, we explore in the present work the electronic and magnetic properties of Sc-doped $\mathrm{ZnO}$ thin films by first principles calculations. Note that nonmagnetic Sc can induce a ferromagnetic ordering in an oxide host due to unfilled $3 d$ states.

All our calculations are carried out within the framework of the spin-density functional theory using the projected augmented wave (PAW) method $^{15}$ as implemented in the Vienna Ab initio Simulation Package. ${ }^{16}$ A plane wave basis set and PAW potentials with the valence states $3 d^{10}$ and $4 s^{2}$ for $\mathrm{Zn}, 2 s^{2}$ and $2 p^{4}$ for $\mathrm{O}$, and $3 p^{6}, 4 s^{2}$, and $3 d^{1}$ for Sc are employed. An energy cut-off of $520 \mathrm{eV}$ for the planewave expansion of the PAWs is used. The exchangecorrelation interaction is treated in the generalized gradient approximation (GGA) using the parametrization of Perdew, Burke, and Ernzerhof. ${ }^{17}$ Moreover, we have applied the rotationally invariant version of the GGA $+U$ method introduced by Liechtenstein et al. ${ }^{18}$ The on-site Coulomb interaction, $U$, and on-site exchange interaction, $J$, are applied to the $d$ orbitals of $\mathrm{Zn}$ and $\mathrm{Sc}$ ( $7 \mathrm{eV}$ and $1 \mathrm{eV}$, respectively). Geometry optimization is performed by conjugate-gradient minimization of the Hellmann-Feynman forces on the atoms and the stresses in the unit cell. Atomic coordinates and axial ratios have been relaxed for different volumes of the unit cell. Convergence is assumed for an energy difference between two successive iterations less than $10^{-6} \mathrm{eV}$ per unit cell and forces on the atoms less than $0.01 \mathrm{eV} / \AA$. All Brillouin zone integrations are performed with a Monkhorst-Pack mesh. ${ }^{19}$ Meshes of $6 \times 4 \times 1$ and $8 \times 6 \times 2$ points are found to be sufficient for the geometry optimization and electronic structure calculations, respectively. 
We construct the $\mathrm{ZnO}$ thin film from the optimized wurtzite bulk $\mathrm{ZnO}$ structure with lattice parameters $a=3.29 \AA$ and $c=5.30 \AA$. The experimental values $(a=3.25 \AA$ and $c=5.20 \AA$, Ref. 20) are overestimated by $1.2 \%$ and $2.0 \%$, respectively. The $\mathrm{ZnO}$ thin film is modeled by a $(1 \times 2)$ seven-layer slab supercell in [11 20$]$ orientation, as shown in Fig. 1, containing 28 formula units. The top and bottom surfaces are separated from other slabs by a vacuum region of $10 \AA$ thickness. Our supercell takes into consideration surface effects which are neglected in bulk calculations. ${ }^{21}$ Note that in our model, the top and bottom of the slab are equivalent by symmetry since the substitution of $\mathrm{Zn}$ by Sc atoms is done on both sides of the slab. The energetically preferable sites for the Sc dopant are determined by calculating the total energy of substitutional Sc in the surface and subsurface layers. The subsurface site is $0.99 \mathrm{eV}$ lower in energy than the surface site.

In order to investigate the magnetic interaction and the preferred distance between two Sc atoms, we substitute two $\mathrm{Zn}$ atoms by Sc atoms on the top and bottom of the slab, giving rise to a doping level of $4 / 28(\sim 14 \%)$. We will study the four configurations shown in Fig. 1. To determine the magnetic state, the total energy is calculated while the spin of the two Sc atoms is set parallel or antiparallel, representing ferromagnetism (FM) or antiferromagnetism (AFM). The energy difference $\Delta E=E_{\mathrm{AFM}}-E_{\mathrm{FM}}$ (per $\mathrm{Sc}$ ) indicates the magnetic ground state of the system, where positive $\Delta E$ corresponds to FM.

We start with an analysis of the magnetic coupling between a pair of Sc atoms in the configurations displayed in Fig. 1. In configuration $\mathrm{C} 1$, two $\mathrm{Zn}$ atoms are replaced by Sc at nearest-neighbor sites in the surface layer of the slab (distance $3.37 \AA$ ). In configuration $\mathrm{C} 2$, two $\mathrm{Zn}$ atoms are substituted by $\mathrm{Sc}$ in the surface layer at a distance of $5.7 \AA$. Configuration $\mathrm{C} 3$ is obtained by replacing two $\mathrm{Zn}$ atoms by Sc impurities at nearest-neighbor sites in the surface and sub-
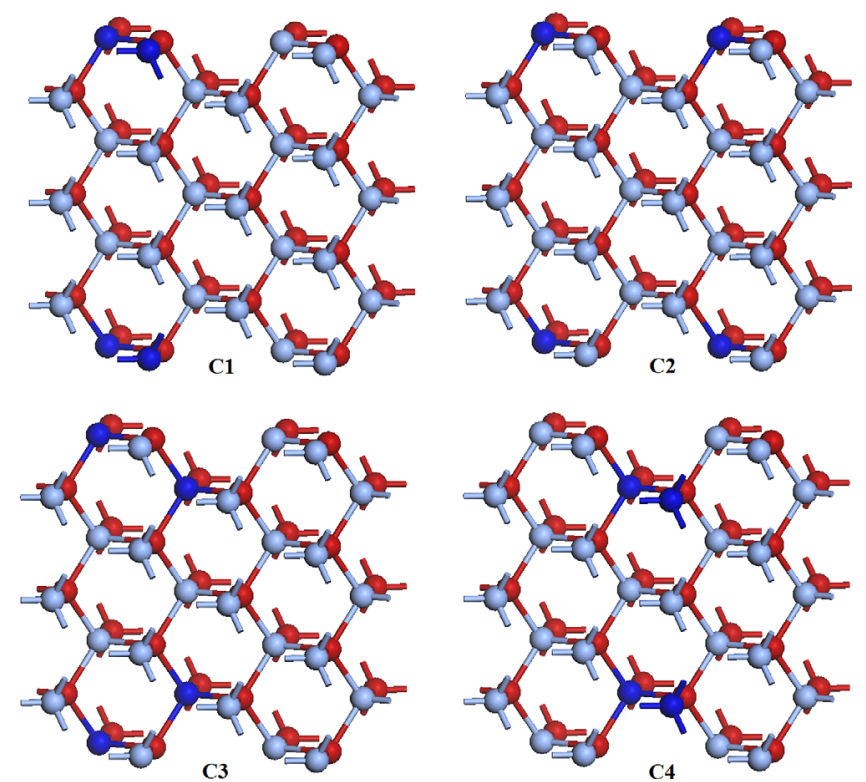

FIG. 1. Side views of four configurations with two substitutional Sc atoms in a $(11 \overline{2} 0)$ surface slab with seven $\mathrm{ZnO}$ layers. The light blue (light gray) spheres are $\mathrm{Zn}$ atoms, the red (gray) are $\mathrm{O}$ atoms, and the blue (dark gray) are Sc. surface layers (distance $3.38 \AA$ ). Finally, in configuration C4 two $\mathrm{Zn}$ atoms are replaced by $\mathrm{Sc}$ in the subsurface layer at a distance of $3.41 \AA$.

We find that configuration $\mathrm{C} 1$ shows a FM ground state (AFM state is $0.88 \mathrm{meV}$ higher in energy). The $\mathrm{Zn}-\mathrm{O}$ and $\mathrm{Sc}-$ $\mathrm{O}$ bond lengths are different at the surface and in the bulk. The $\mathrm{Zn}-\mathrm{O}$ bond length along the [0001] and [1100] directions changes from the initial $2.01 \AA$ and $2.00 \AA$, to $1.91 \AA$ and $1.93 \AA$, respectively. The analogous Sc-O bond length decreases from $2.01 \AA$ and $2.00 \AA$, to $1.97 \AA$ and $1.97 \AA$, respectively. In contrast, the $\mathrm{Sc}_{1}-\mathrm{Sc}_{2}$ bond length increases during the relaxation from $2.21 \AA$ to $3.37 \AA$. In the FM state of configuration $\mathrm{C} 1$, the magnetic moments are mainly localized on the surface Sc atoms, with a local magnetic moment of $0.065 \mu_{B}$. The magnetic moments are almost exclusively due to the Sc $3 d$ states. In contrast, magnetic moments in bulk Sc-doped $\mathrm{ZnO}$ with $\mathrm{Zn}$ vacancies are mainly due to the $2 p$ gap states of the $\mathrm{O}$ atoms around the $\mathrm{Zn}$ vacancies. ${ }^{22}$ Note that the $\mathrm{O}$ atom in the surface layer of our slab has a very small local magnetic moment of $0.005 \mu_{B}$.

In configuration $\mathrm{C} 3$, the $\mathrm{FM}$ state is $0.63 \mathrm{meV}$ lower in energy than the AFM state. The $\mathrm{Sc}_{1}-\mathrm{Sc}_{2}$ distance changes from the initial $3.25 \AA$ to $3.407 \AA$ during the relaxation. The $\mathrm{Sc}_{1}-\mathrm{O}$ and $\mathrm{Sc}_{2}-\mathrm{O}$ bond lengths are $1.94 \AA$ and $2.03 \AA$, in the surface and subsurface layer, respectively. The difference to configuration $\mathrm{C} 1$ is that the $\mathrm{Zn}$ atoms next to $\mathrm{Sc}$ atoms in the surface layer are magnetized with a moment of $0.105 \mu_{B}$. We also find that the $\mathrm{O}$ neighbors of $\mathrm{Sc}$ atoms in the surface and subsurface layers show smaller magnetic moments of $0.020 \mu_{B}$ and $0.018 \mu_{B}$, while $\mathrm{Sc}_{1}$ and $\mathrm{Sc}_{2}$ carry weak moments of $0.005 \mu_{B}$ and $0.001 \mu_{B}$. The FM state of configuration $\mathrm{C} 3$ is $1.67 \mathrm{eV}$ lower in energy than the FM state of configuration $\mathrm{C} 1$. The Sc-O distances are similar in $\mathrm{C} 1$ and $\mathrm{C} 3$, but the $\mathrm{Sc}_{1}-\mathrm{Sc}_{2}$ distances are rather different. Configurations $\mathrm{C} 2$ and $\mathrm{C} 4$ do not show any magnetization.

To check the convergence of our calculations with respect to the supercell size, we compare fully relaxed supercells with different thicknesses along the [11̄20] direction.

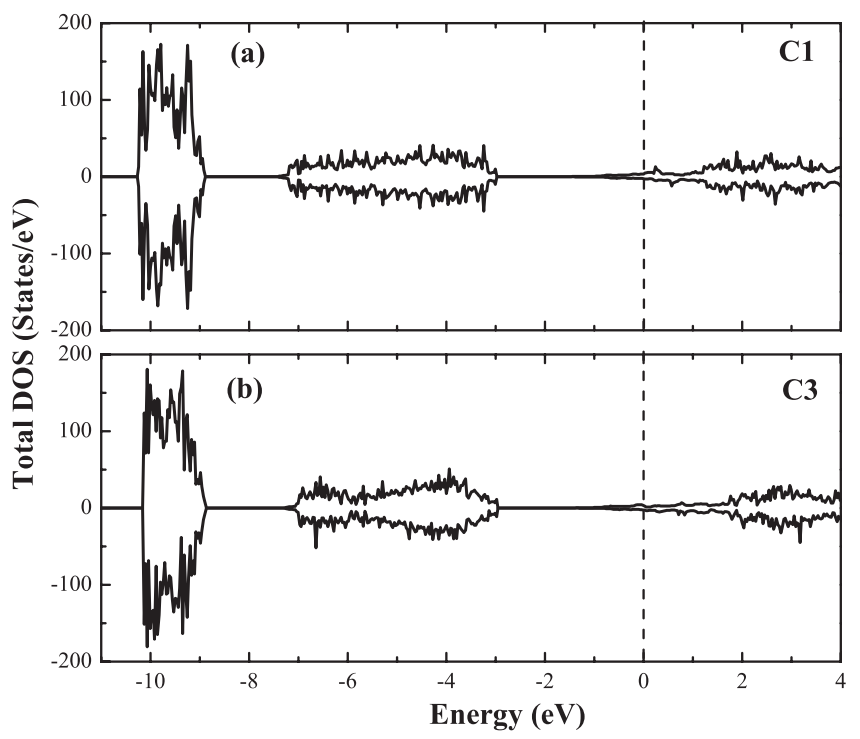

FIG. 2. Total spin-polarized DOS for configurations C1 and C3. Spin up and spin down correspond to positive and negative values, respectively. The vertical dashed line denotes the Fermi level. 

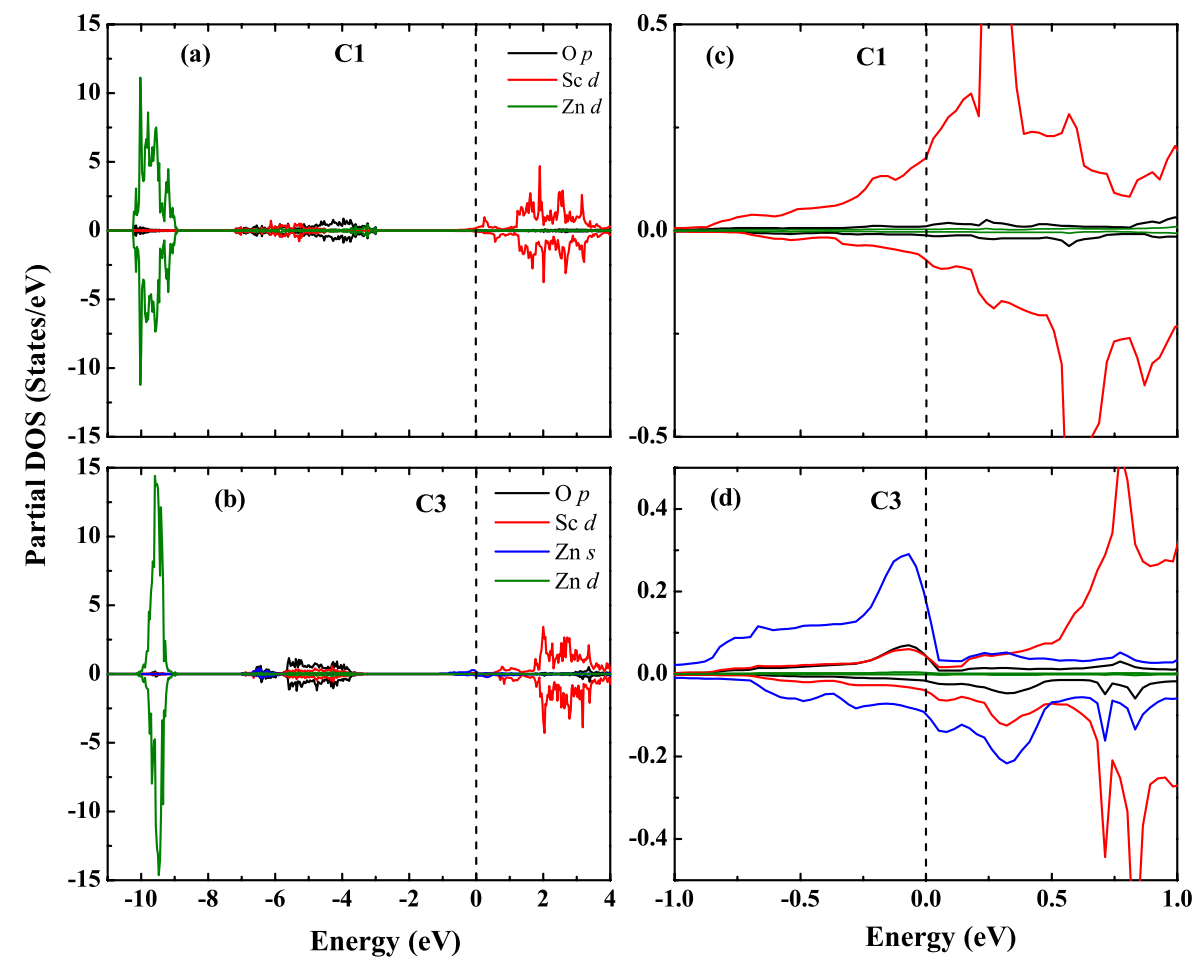

FIG. 3. Sc, Zn, and O partial DOSs of configurations (a) $\mathrm{C} 1$ and (b) $\mathrm{C} 3$. The local $\ell$-projected DOS is magnified over the range -1 to $1 \mathrm{eV}$ in (c) and (d).
We address slabs of 9 layers and 11 layers with a total of 72 and 88 atoms, respectively. We find that FM is more favorable than AFM with energy differences of $0.66 \mathrm{meV}$. When the thickness increases beyond 7 layers, the magnetic moment shows no significant change. The same is observed for transition metal doping of $\mathrm{ZnO} .^{23-25}$

To elucidate the role of the Sc-doping on the electronic structure and to obtain information on the roles of the individual orbitals and atoms, we address the $\mathrm{GGA}+U$ density of states (DOSs). Figures 2 and 3 provide the total and partial DOSs of the FM calculations for configurations $\mathrm{C} 1$ and $\mathrm{C} 3$. They show common features: (i) The $\mathrm{Zn}$ states are localized between -10 and $-9 \mathrm{eV}$, well below the $\mathrm{O} 2 p$ states. (ii) The occupied $\mathrm{O} 2 p$ states appear between -7 and $-3 \mathrm{eV}$ showing some hybridization with the $\mathrm{Zn} 4 d$ states. (iii) $\mathrm{Sc}$ contributions to the valence states are small. It is interesting to note that the Sc doping maintains the semiconducting nature of $\mathrm{ZnO}$ and introduces impurity states at the bottom of the conduction band (both for the majority and minority spin channels). However, in the vicinity of the Fermi energy the electronic states of the two configurations show different behaviors. The DOS of $\mathrm{C} 1$ in Fig. 3(a) shows that the Sc $3 d$ states dominate at the Fermi energy. We observe that the $\mathrm{O}$ $2 p$ orbital is hybridized slightly with the Sc $3 d$ orbital, see Fig. 3(c), and O states appear at the Fermi level. Nonetheless, only a very weak polarization affects the surface $\mathrm{O}$ atoms. In the case of configuration C3, see Fig. 3(d), we notice a simultaneous hybridization between the $\mathrm{Zn} 4 s$, O $2 p$, and Sc $3 d$ states around the Fermi level.

The spin density is calculated for configurations $\mathrm{C} 1$ and C3, and displayed in Fig. 4 as isosurface plots. ${ }^{26,27}$ Figure 4(a) shows that the spin-polarized electrons are localized at

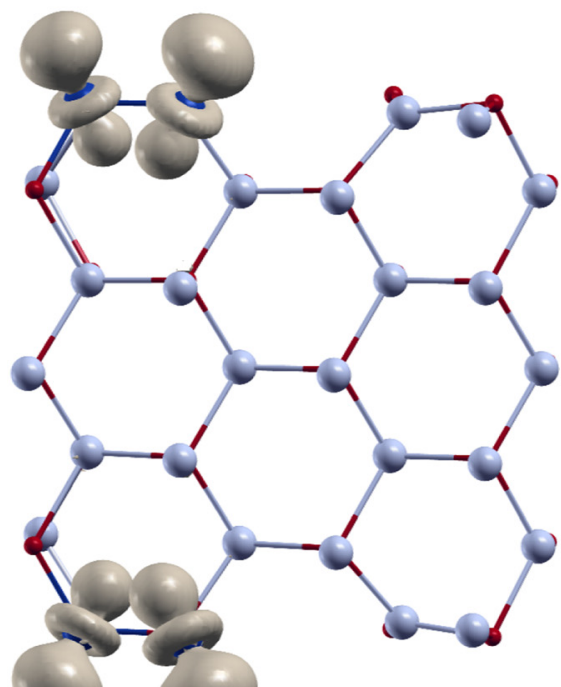

(a)

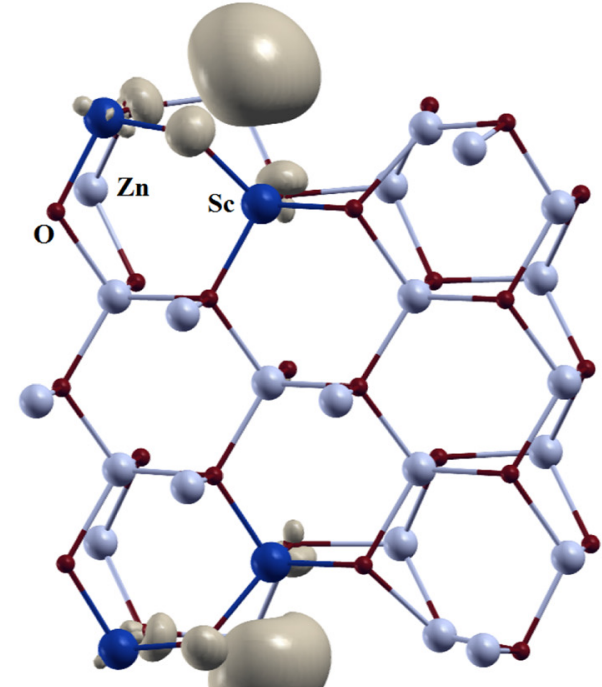

(b)
FIG. 4. Isosurface spin density plot for configurations (a) $\mathrm{C} 1$ and (b) $\mathrm{C} 3$ at an isovalue of 0.01 electron $/ \AA^{3}$. 
the Sc sites in the surface layer. The spin density distribution has a $d$ like shape, which indicates that the polarization is induced by the $d$ orbitals and thus confirms a local nature of the magnetic moments in the system. Turning to configuration C3 in Fig. 4(b), we find that the magnetization is carried mainly by the $\mathrm{Zn} s$ orbitals due to the $s$-like shape of the isosurface.

In summary, we have performed ab-initio calculations to investigate the electronic structure and magnetic properties of Sc-doped $\mathrm{ZnO}$ thin films. We conclude that even though Sc originally is not magnetic, it can induce ferromagnetism in $\mathrm{ZnO}(11 \overline{2} 0)$ thin films, in agreement with experiments. However, we have identified only two substitution patterns which result in spin polarization. In both cases, ferromagnetism is energetically preferred. Configuration C3 is more stable than configuration $\mathrm{C} 1$, in which the spin polarization is carried by the Sc $3 d$ states (as to be expected for transition metal doped $\mathrm{ZnO}$ ). It is found that the spin polarization in configuration $\mathrm{C} 3$ is connected to a strong hybridization between the Sc $3 d$ states and the $\mathrm{ZnO}$ host. While so far it has been assumed that mainly the transition metal $d$ orbitals are responsible for the magnetism in doped $\mathrm{ZnO}$ thin films, our results for Sc doping draw a different picture. The spin polarization affects highly delocalized hybrid states (comprising $\mathrm{Sc} d$, O $p$, and $\mathrm{Zn} s$ orbitals) formed at the surface of the $\mathrm{ZnO}$ thin film. In a realistic experiment, the doping process should result in a random configuration of the impurities of C1 and C3 type. Note, however, that configuration C3 is most likely to occur due to its lower energy. Our study shows that the magnetism in Sc-doped $\mathrm{ZnO}$ probably possesses an itinerant character.

${ }^{1}$ H. Ohno, Science 281, 951 (1998).

${ }^{2}$ Y. Matsumoto, M. Murakami, T. Shono, T. Hasegawa, T. Fukumura, M. Kawasaki, P. Ahmet, T. Chikyow, S. Koshihara, and H. Koinuma, Science 291, 854 (2001).

${ }^{3}$ F. Pan, C. Song, X. J. Liu, Y. C. Yang, and F. Zeng, Mater. Sci. Eng. R. 62, 1 (2008).
${ }^{4} \ddot{U}$. Özgür, Y. I. Alivov, C. Liu, A. Teke, M. A. Reshchikov, S. Doan, V. Avrutin, S.-J. Cho, and H. Morko, J. Appl. Phys. 98, 041301 (2005).

${ }^{5}$ T. Dietl, H. Ohno, F. Matsukura, J. Cibert, and D. Ferrand, Science 287, 1019 (2000).

${ }^{6}$ S. B. Ogale, Adv. Mater. 22, 3125 (2010).

${ }^{7}$ K. Sato, L. Bergqvist, J. Kudrnovsk, P. H. Dederichs, O. Eriksson, I. Turek, B. Sanyal, G. Bouzerar, H. Katayama-Yoshida, V. A. Dinh, T. Fukushima, H. Kizaki, and R. Zeller, Rev. Mod. Phys. 82, 1633 (2010).

${ }^{8}$ I. Bantounas, S. Goumri-Said, M. B. Kanoun, A. Manchon, I. Roqan, and U. Schwingenschlögl, J. Appl. Phys. 109, 083929 (2011).

${ }^{9}$ L. H. Ye, A. J. Freeman, and B. Delley, Phys. Rev. B 73, 033203 (2006).

${ }^{10}$ D. B. Buchholz, R. P. H. Chang, J. H. Song, and J. B. Ketterson, Appl. Phys. Lett. 87, 082504 (2005).

${ }^{11}$ T. S. Herng, D.-C. Qi, T. Berlijn, J. B. Yi, K. S. Yang, Y. Dai, Y. P. Feng, I. Santoso, C. Sanchez-Hanke, X.Y. Gao, A. T. S. Wee, W. Ku, J. Ding, and A. Rusydi, Phys. Rev. Lett. 105, 207201 (2010).

${ }^{12}$ K. Osuch, E. B. Lombardi, and W. Gebicki, Phys. Rev. B 73, 075202 (2006).

${ }^{13}$ M. Venkatesan, C. B. Fitzgerald, J. G. Lunney, and J. M. D. Coey, Phys. Rev. Lett. 93, 177206 (2004).

${ }^{14}$ C.-H. Chien, S. H. Chiou, G. Y. Guo, and Y.-D. Yao, J. Magn. Magn. Mater. 282, 275 (2004).

${ }^{15}$ P. E. Blöchl, Phys. Rev. B 50, 17953 (1994).

${ }^{16}$ G. Kresse and J. Furthmüller, Comput. Mater. Sci. 6, 15 (1996).

${ }^{17}$ J. P. Perdew, K. Burke, and M. Ernzerhof, Phys. Rev. Lett. 77, 3865 (1996).

${ }^{18}$ A. I. Liechtenstein, V. I. Anisimov, and J. Zaanen, Phys. Rev. B 52, R5467 (1995).

${ }^{19}$ H. J. Monkhorst and J. D. Pack, Phys. Rev. B 13, 5188 (1976).

${ }^{20}$ E. H. Kisi and M. M. Elcombe, Acta Crystallogr. C 45, 1867 (1989).

${ }^{21}$ S. Goumri-Said and M. B. Kanoun, Phys. Lett. A 374, 3977 (2010).

${ }^{22}$ M. B. Kanoun, S. Goumri-Said, U. Schwingenschlögl, and A. Manchon, Chem. Phys. Lett. 532, 96 (2012).

${ }^{23}$ Q. Wang and P. Jena, Appl. Phys. Lett. 84, 4170 (2004).

${ }^{24}$ Q. Wang, Q. Sun, P. Jena, Z. Hu, R. Note, and Y. Kawazoe, Appl. Phys. Lett. 91, 063116 (2007).

${ }^{25}$ Q. Wang, Q. Sun, P. Jena, and Y. Kawazoe, Phys. Rev. B 79, 115407 (2009).

${ }^{26}$ The three-dimensional spin-density is obtained by the full-potential (linearized) augmented plane waves plus local orbitals method ${ }^{27}$ in the GGAPBE approximation. The self-consistent procedure is carried out with $\mathrm{R}_{M T} \times \mathrm{K}_{\text {max }}=8$ and the Brillouin zone integrations are performed with the special k-points method over a $8 \times 6 \times 1$ Monkhorst-Pack mesh. ${ }^{19}$

${ }^{27}$ P. Blaha, K. Schwarz, G. K. H. Madsen, D. Kvasnicka, and J. Luitz, WIEN2k, An Augmented Plane Wave + Local Orbitals Program for Calculating Crystal Properties (TU Vienna, Vienna, 2001). 